\title{
ANÁLISIS DE LA FUNDAMENTACIÓN DIDÁCTICA DE LOS LIBROS DE TEXTO DE CONOCIMIENTO DEL MEDIO SOCIAL Y CULTURAL
}

\section{(ANALYSIS OF THE DIDACTIC BASIS OF SOCIAL SCIENCES TEXTBOOKS)}

\author{
Gabriel Travé \\ Jesús Estepa \\ Universidad de Huelva \\ Juan Delval \\ Universidad Autónoma de Madrid
}

DOI: $10.5944 / e d u c X X 1.11831$

\section{Cómo referenciar este artículo/How to reference this article:}

Travé, G.; Estepa, J. y Delval, J. (2017). Análisis de la fundamentación didáctica de los libros de texto de conocimiento del medio social y cultural. Educación XX1, 20(1), 319-338, doi: 10.5944/ educXX1.11831

Travé, G.; Estepa, J. \& Delval, J. (2017). Análisis de la fundamentación didáctica de los libros de texto de conocimiento del medio social y cultural. [Analysis of the didactic basis of social sciences textbooks]. Educación XX1, 20(1), 319-338, doi: 10.5944/educXX1.11831

\section{RESUMEN}

Este artículo focaliza la atención sobre los materiales curriculares con el objetivo de analizar la fundamentación didáctica que explícita o tácitamente presentan los libros de texto de Conocimiento del Medio. Se han seleccionado los temas referidos a sociedades actuales e históricas y actividades económicas en Primaria, a través del estudio de las editoriales mayoritarias del panorama español y de entrevistas al profesorado que las utiliza. Entre las conclusiones, se identifican en los textos obstáculos epistemológicos, axiológicos y psicológicos que dificultan la comprensión por el alumnado de las complejas sociedades actuales y de las históricas, constatando la inadecuación de los textos a los avances producidos por la investigación didáctica y psicológica.

\section{PALABRAS CLAVE}

Libro de texto; enseñanza ciencias sociales; concepciones profesorado; fines de la educación. 


\section{ABSTRACT}

The article is focused on the study of curriculum materials with the aim to analyze the didactic basis that Social and Natural Science textbooks present explicitly or implicitly. Two studies have been undertaken, the first one related to textbooks analysis and the second based on a number of interviews with the teachers who use them. Among the findings, epistemological, axiological and psychological obstacles have been identified in the texts, hindering students' understanding of complex current and historical societies, noting the inadequacy of the texts to the progress made by educational and psychological research.

\section{KEYWORDS}

Textbook; social sciences; teacher attitudes; education objectives.

\section{INTRODUCCIÓN}

Una de nuestras principales líneas de investigación está relacionada con la contribución de los libros de texto a la comprensión y actuación del alumnado en el medio social y cultural. Por ello, a continuación tratamos someramente algunos referentes que ilustran el estado de la investigación acerca de la construcción del pensamiento social, histórico y económico de los estudiantes en Primaria y el estudio de los materiales curriculares con contenido en esas tres dimensiones de la realidad social.

Los trabajos sobre concepciones relativas a las sociedades actuales e históricas han puesto de manifiesto la importancia de este campo de conocimiento (Estepa, 2003; Rodríguez y Carretero, 2012). Las conclusiones son unánimes al comprobar los obstáculos que atraviesan los alumnos en la comprensión de las nociones temporales (cronología, orientación, tiempo histórico y social...), así como la consideración finalista que otorgan a la evolución histórica. Sin embargo, estas y otras investigaciones indican que las dificultades en el aprendizaje de la Historia y el tiempo histórico están más relacionadas con su enseñanza que con las capacidades del alumnado, pudiendo obtenerse mejores resultados si se modifica la metodología didáctica, los materiales y recursos para la facilitación del aprendizaje y el propio contenido a enseñar (Levesque, 2008; Santisteban, 2007).

Los estudios relacionados con la alfabetización económica de los alumnos, por otra parte, constatan las dificultades de comprensión infantil del mundo económico respecto a las relaciones de intercambio, de producción, con el medio y de desigualdad (Armento, 2003; Delval, 2013; Díez, 2012; 
Travé y Delval, 2009). Las conclusiones acerca de la investigación sobre esta problemática han demostrado que la construcción del pensamiento avanza, entre otros, con la edad, las experiencias económicas vividas y la instrucción recibida (Miller y Vanfossen, 2008; Posnanski, Schug, Schmitt, 2006).

En cuanto a las investigaciones en las que se procede al análisis didáctico de los materiales curriculares y de los libros de texto con contenido social destacan, entre otros, los trabajos de Atienza y Van Dijk (2010), Blanco (2007), Caba y López Atxurra (2005), Hernández (2012), Nicholls (2003), Travé y Pozuelos (2008), Valls (2007). En estos estudios, centrados en el libro de texto, se constata principalmente la escasez de actividades abiertas en relación con el medio social y cultural, frente al predominio de los simples ejercicios de repaso de contenidos del libro; la presencia de numerosos errores e incorrecciones científicas y didácticas; la promoción de un modelo de enseñanza tradicional; en fin, la utilización de procesos de maquillaje educativo entre el proyecto editorial, adaptado a las exigencias del currículum oficial, y el texto editado para el alumnado.

\section{MÉTODO}

Este trabajo forma parte de un proyecto más amplio cuya finalidad consiste en desarrollar un análisis didáctico de los manuales escolares de Conocimiento del Medio social y cultural de Educación Primaria y contrastarlo con las declaraciones de los docentes relacionadas con su utilización.

\section{INSTRUMENTOS}

El análisis de los libros de texto se implementa a través de una ficha o parrilla de codificación y recogida de información, que integra dos partes: una cabecera, para reseñar los datos del material (tipo, etapa, formato, título, autores, edición, etc.); y otra, en la que mediante categorías e indicadores se extraen datos del material sometido a análisis, incluyendo párrafos del texto que avalen el indicador seleccionado. Mientras el instrumento empleado en el estudio 2, ha sido la entrevista colectiva de 6-7 profesores-as, de carácter semiestructurada y compuesta por 25 preguntas, con una duración aproximada de 2 horas y que fue administrada a una muestra de 5 centros de Educación pública, 1 Centro de Profesorado y 1 Movimiento de Renovación Educativa.

La ficha y las entrevistas se han elaborado a partir del instrumento de análisis de materiales y desarrollo de la enseñanza -AMADE—, que es común para el tratamiento de los datos de ambos estudios (Travé et al., 2013). 
El instrumento AMADE fue diseñado, validado y experimentado siguiendo otros trabajos anteriores acerca de los obstáculos del profesorado para desarrollar procesos de investigación escolar ${ }^{2}$ (Pozuelos, Travé y Cañal, 2007 y 2010). Su elaboración se organiza en torno a un sistema de categorías e indicadores, siguiendo entre otros a Mckernan (1999) y Simons (2011), y se concreta en un formato válido para codificar datos procedentes del análisis de distintas fuentes (documentos, cuestionarios, entrevistas, observaciones, etc). Esta matriz de codificación incluye las categorías: epistemológica, axiológica, psicológica, elementos curriculares, diseño de la enseñanza y desarrollo profesional, aunque en este trabajo se estudian solo la tres primeras (Tabla 1).

El proceso de diseño, validación y administración de los instrumentos de investigación ha sido paralelo y simultáneo en los dos estudios:

- Fase de diseño: confección de los instrumentos (ficha de análisis y entrevista) siguiendo las categorías de la investigación.

- Fase de validación: la versión preliminar de la validación de ambos instrumentos requirió la técnica de juicio de expertos, por siete profesores de diversas universidades españolas y ocho maestros en activo. Esta prueba puso de manifiesto la existencia de una serie de obstáculos similares a los descritos por Estepa, et al. (2011). Así, por una parte, se detectaron obstáculos de índole conceptual, basados en las desiguales representaciones cognitivas del grupo de investigadores respecto a las nociones estudiadas; y, por otro, de carácter metodológico, referidos a la búsqueda de consenso para asignar indicadores que no se correspondían con el hecho analizado. Para ello se definieron con precisión mediante un trabajo continuado y colaborativo del grupo de investigadores, las opciones coherentes con el paradigma didáctico actual (Ind.A), el indicador de transición (Ind.B) y el más próximo a la enseñanza convencional (Ind.C). En caso de inadecuación de los indicadores al texto, se requiere del investigador la formulación de una nueva opción (Ind.D).

- Fase de administración de los diversos instrumentos: el criterio de selección de la muestra, en el estudio 1, se definió en función de los manuales de uso y difusión mayoritarios en la Comunidad andaluza. La muestra está constituida por los libros de texto de $1 .^{\circ}$ a $6 .^{\circ}$ de Educación Primaria de las dos editoriales mayoritarias, publicados principalmente en el año $2009^{3}$. El análisis se ha efectuado examinando una muestra de 45 temas relativos a la enseñanza de contenido social, histórico y económico. Mientras para las declaraciones del profesorado, se seleccionaron cinco centros ubicados 
en zonas socioecómicas favorecidas y desfavorecidas de titularidad pública de Huelva capital y provincia. El tratamiento de datos de ambos estudios se ha procesado con el programa ATLAS.ti en su versión 6.2, y para la cita de contenidos procedentes de este análisis hemos atendido a la nomenclatura que se observa en el siguiente ejemplo: "Las tareas tienen una visión muy práctica y los alumnos están más motivados» $(4: 37,248: 250$, D5). De tal manera, los códigos utilizados tienen el siguiente significado: 4:37, número de recurso (documento): número de cita en dicho documento; 248:250, párrafo donde se inicia la cita: párrafo donde termina la cita; D5, docente que realiza la afirmación.

Tabla 1.

Sistema de categorías y contenidos de investigación

\begin{tabular}{l|l}
\hline \multicolumn{1}{c|}{ Categorías } & \multicolumn{1}{c}{ Contenidos } \\
\hline $\begin{array}{l}\text { Categoría 1. } \\
\text { Fundamentación epistemológica }\end{array}$ & $\begin{array}{l}\text { Indaga acerca del conocimiento transmitido } \\
\text { por el material y la relación establecida entre } \\
\text { el conocimiento científico, cotidiano y esco- } \\
\text { lar sobre la realidad social y natural. }\end{array}$ \\
\hline $\begin{array}{l}\text { Categoría 2. } \\
\text { Fundamentación axiológica }\end{array}$ & $\begin{array}{l}\text { Examina las finalidades y los valores que } \\
\text { transmiten los materiales de Conocimiento } \\
\text { del Medio. }\end{array}$ \\
\hline Categoría 3. & $\begin{array}{l}\text { Estudia el tipo de aprendizaje que favorece } \\
\text { el material, así como la función que tienen } \\
\text { los conocimientos previos del alumnado. }\end{array}$ \\
Fundamentación psicológica
\end{tabular}

\section{RESULTADOS}

El sistema de categorías e indicadores comentado anteriormente y otras observaciones y procedimientos de análisis libre basados en el Proyecto Investigando Nuestro Mundo (6-12) ${ }^{4}$, servirán para presentar los resultados, además de la discusión de los mismos.

\section{Fundamentación epistemológica}

Diversos autores han estudiado las teorías epistemológicas que inciden en el marco educativo (Cañal, Travé y Pozuelos, 2011; Porlán y Rivero, 1998; Solaz-Portolés, 2010). 
La tradición positivista concibe el conocimiento científico como absoluto, verdadero y generalizable; el conocimiento cotidiano del alumno con el calificativo de erróneo y desechable y el conocimiento escolar como una versión reducida y adaptada al alumnado del conocimiento científico. El paradigma crítico, en contraposición, considera que la ciencia es una construcción humana y, por tanto, relativa y cambiante. Este enfoque epistemológico, denominado por Fourez (2008) socioconstructivista, considera que el conocimiento cotidiano sirve de catalizador para construir cualquier saber y que el conocimiento escolar se produce en la interacción del conocimiento científico y el conocimiento cotidiano.

Tomando como referencia estas posiciones epistemológicas extremas, se han analizado los materiales curriculares, obteniendo que el indicador mayoritario en los temas relacionados con las sociedades actuales e históricas y las actividades económicas es el Ind.B (El conocimiento escolar es una copia reducida del conocimiento científico). Así, en la unidad de $6 .^{\circ} \mathrm{de}$ Primaria titulada La Edad Contemporánea, en relación con Andalucía en el siglo XIX, se indica que «Durante el transcurso del siglo XIX, tuvieron lugar en Andalucía acontecimentos muy destacados: guerra de la Independencia...; reinado de Fernando VII...; reinado de Isabel II...; reinado de Alfonso XII»(Gómez, Valbuena y Brotons, 2009a, p. 210).

Como puede observarse se trata de un conocimiento básicamente descriptivo, en el que se establecen pocas relaciones explícitas, el cambio se señala únicamente en relación con el conocimiento histórico de carácter político y los objetos de estudio se formulan como temas muy próximos al conocimiento científico, obviando la historia social de la época, más cercana y comprensible para el conocimiento cotidiano.

Sin embargo, de forma minoritaria en los libros de texto analizados se observa otro enfoque, Ind.D (El conocimiento escolar indica un mayor empleo del conocimiento cotidiano), así, en relación con las actividades económica, Gómez, et al. (2009a, p. 169) presentan las preguntas siguientes: ¿qué crees que vende una agencia de viajes?, ¿por qué no se construye un gran centro comercial en una pequeña aldea?, ¿cuántos empleados se necesitan en un quiosco de prensa y en un gran centro comercial?, ¿qué te parece más importante, una gran empresa o una empresa pequeña? Tales cuestiones, si bien establecen algunas relaciones entre conocimiento escolar, cotidiano y científico, aprovechando el interés del alumnado por los temas económicos, no tienen continuidad en el desarrollo del texto, dedicado nuevamente a los sectores de producción.

Este indicador minoritario está condicionado por la edad del alumnado y el contenido. Así, en primer ciclo se concede mayor importancia al 
conocimiento cotidiano, con títulos de temas como: Tenemos una familia, ¿Cómo es tu casa?, Viajamos, Así somos, Vivimos juntos, El paso del tiempo (1. ${ }^{\circ}$ y $2 .^{\circ}$ curso, de Santillana). También se observa en la unidad La calle de $1 .^{\circ}$ (Herrero y Martín, 2010a), pidiendo al alumnado que complete la dirección de su domicilio. Igualmente se concede este tratamiento más cotidiano, dependiendo del contenido abordado, sobre todo cuando se trata de temáticas sociales y económicas, como por ejemplo: vivimos en comunidad y exploramos la localidad (Gómez et al., 2009a).

Las declaraciones del profesorado muestran otra tipología epistemológica adoptada en función de los factores contextuales del centro escolar, próxima al Ind.A. (El conocimiento escolar es la interacción del conocimiento cotidiano y científico). Así, al ser cuestionados por el tipo de materiales que utilizan en Conocimiento del Medio, los docentes del CEIP Andalucía (Huelva) realizan las siguientes afirmaciones:

No utilizamos libros de texto, porque en un entorno desfavorecido los alumnos-as no lo siguen y se aburren. Elaboramos unidades o proyectos de investigación (4:1,009:009, D4).

El contenido de los libros está más estructurado y si nosotros trabajamos con un proyecto abrimos mucho más el tema (4:70 051:051, D3).

Por ejemplo, el proyecto de la tienda, donde utilizamos los productos que se agotan en casa para trabajar desde normas de cortesía, de higiene, de economía (como comprar y vender). Hay ofertas de la semana y se hacen actividades a partir de la tienda, como hacer una paella (4:34 y 36, 232:244, D2).

Las tareas tienen una visión muy práctica y los alumnos están más motivados (4:37, 248:250, D5).

En este barrio hay muchas familias que viven de los mercadillos y un proyecto que salió muy bonito fue montar un mercadillo, para ello pedimos permiso al ayuntamiento, medimos el terreno, elaboramos las cosas que se iban a vender, llevamos una hoja de contabilidad, un inventario. Todo globalizado (4:39, 252:252, D5).

Recapitulando, los datos del análisis de los libros de texto en cuanto a la epistemología que transmiten indican mayoritariamente que el conocimiento escolar es una copia reducida del conocimiento científico, con algunas pequeñas matizaciones en cuanto a la edad y al contenido; sin embargo, en el ejemplo de la entrevista, se observa otra forma de epistemología basada 
en proyectos de investigación implementados en el centro. En este caso los docentes sostienen que el conocimiento escolar está constituido por el conocimiento cotidiano enriquecido con referencias de conocimiento científico.

\section{Fundamentación axiológica}

La determinación de las finalidades educativas ha sido una temática pensada y repensada a lo largo del tiempo (Domínguez y Feito 2007; Niemi y Junn, 1998; Osborne, 1991; Pagès y Santisteban, 2011). En el caso de la enseñanza de las Ciencias Sociales, traemos a colación, en primer lugar, la propuesta de Benejam (2015) que clasifica las finalidades en cultural, práctica y crítica o reflexiva, dependiendo de la manera de concebir los valores que se pretenden transmitir por el material. Para el caso de la enseñanza de la Historia, García y Leduc (2003) consideran que su conocimiento permite el desarrollo de una ciudadanía democrática participativa y una socialización crítica. Además, Prats y Santacana (2011) atribuyen a la enseñanza de esta materia las finalidades referidas a la comprensión del presente, al desarrollo de facultades intelectuales, al enriquecimiento de otros temas de la vida y a la adquisición de conciencia social sobre problemas relevantes.

Las finalidades de la alfabetización económica de la población fomentan, para Miller y Vanfossen (2008), la formación de los ciudadanos a nivel personal y público, y constituyen un aspecto necesario para el desenvolvimiento de las sociedades democráticas, incrementando el desarrollo económico de los países. En definitiva, la meta principal de la educación social, económica e histórica, es la comprensión de los problemas de nuestro mundo y la formación para el ejercicio de una ciudadanía responsable, activa, global y crítica (Estepa, 2012; Houser y Kuzmic, 2001; McDonough y Feinberg, 2003; Sobejano y Torres, 2009).

Los datos del análisis de los manuales escolares indican que, en algunos temas, preferentemente de los primeros ciclos, se establece un apartado de educación en valores, como en Viajamos de 1. (Alzu y López Sáez, 2008), en el que se indica «los trenes y los autobuses son para uso de todos ¿Cómo puedes ayudar a cuidarlos?» (p.136) En otros temas, en este caso de 2. ${ }^{\circ}$ (Alzu y Henao, 2010), al comienzo figura un apartado denominado La frase para pensar, que en el libro del profesor se acompaña de un recuadro de valores para trabajar. También en los libros de $5 .^{\circ}$ y $6 .^{\circ}$ de esta serie (Alzu y Henao, 2009 a y 2009b) se establece un apartado titulado El mundo que queremos, donde se trabajan valores en relación con el tema que se está tratando.

Sin embargo, no hemos encontrado apenas ejemplos de contenidos o actividades «para desarrollar un pensamiento práctico, reflexivo y crítico 
que contribuyan a mejorar la realidad social y natural» (Ind.A), y sí muchos que se plantean la educación en valores como una finalidad complementaria y secundaria. Así, en la unidad de $6 .^{\circ}$ denominada La Edad Contemporánea (Gómez, et al., 2009a, p .212) se establecen 8 contenidos mínimos, siete sobre hechos, informaciones y conceptos y uno de carácter actitudinal que se enuncia como "valoración de la democracia como el sistema político que defiende la igualdad y garantiza nuestros derechos y libertades»; no obstante, en el resumen del tema no se considera que sea un contenido de importancia para que se recoja en el mapa conceptual. Además de eludir paradójicamente toda referencia a la estrategia didáctica de «aprender haciendo» democracia en la escuela.

En cuanto a los valores observados en los temas de actividades económicas, siguen la tónica comentada anteriormente. De este modo, Etxebarría, et al. (2009, p. 149) proponen en el apartado «El mundo que queremos», el ejercicio siguiente sobre el consumo responsable:

Vivimos en un mundo en el que cada vez consumimos más. Para producir tanto se recurre muchas veces a la explotación de niños y adultos, a la contaminación de la naturaleza o a la creación de residuos. Por ello, es necesario que practiquemos un consumo responsable y el reciclado de las cosas. Haced una lista con las medidas que podéis tomar para consumir menos en casa y en el colegio.

El texto presenta el problema del consumo y su repercusión en la explotación de las personas y del medio ambiente. Sin embargo, la actividad propuesta encubre, en gran parte, la hipocresía social al centrar el problema y la solución en las medidas que puedan tomar los propios niños y sus familias, soslayando la responsabilidad de las empresas y de los gobiernos en los desmanes originados por el propio sistema de desarrollo social consumista.

Las declaraciones del profesorado del CEIP El Puntal sobre la finalidad que persiguen los libros de texto muestran asimismo similitudes con los datos del análisis realizado.

Cultura, vocabulario, conocer cosas nuevas... $(2: 57,166: 166, \mathrm{D} 6)$.

Conceptos. Nada más que buscan conceptos (2:58, 167:167, D1).

Las actividades del libro pretenden rellenar huecos, como el Hot Potatoes $^{5}$ de las nuevas tecnologías, actividades que se hacen de primero a sexto $(2: 59,169: 169, \mathrm{D} 1)$. 
El resultado de ambos análisis sobre los temas de actividades económicas y sociedades actuales e históricas de los manuales y de la práctica reflexionada de los profesores, revela que los indicadores más representativos de las finalidades que se persiguen, son el Ind.C «aporta al alumnado los conocimientos necesarios para superar los cursos escolares», y en menor medida, el Ind.B, porque "propone conocimientos culturales y de respeto por el medio", aunque sea en muchas ocasiones a modo de sugerencia, comentario o recomendación en el libro del profesor. Sin embargo, las finalidades práctica y crítica son escasamente tratadas en los textos escolares, precisamente aquellas que promueven el desenvolvimiento del alumnado en la sociedad, mediante la reflexión y la crítica constructiva.

\section{Fundamentación psicológica}

Desde la perspectiva de las teorías constructivistas del aprendizaje, se han realizado grandes aportaciones al campo de la Didáctica de las Ciencias Sociales. Entre otros trabajos que resultan interesantes para nuestro objetivo, podemos citar los estudios de, Delval, et al. (2006); Barton (2008); Carretero y López (2009); Díaz Castorina (2010); García Pérez (2003). Una síntesis de estos trabajos constata que el tipo de aprendizaje que debe generar el material ha de ser significativo y funcional, relacionando las ideas personales con las nuevas informaciones procedentes de diferentes fuentes de información. Asimismo, el punto de partida del aprendizaje contempla en todo momento los conocimientos e intereses del alumnado, que interaccionan a lo largo del proceso de enseñanza y aprendizaje.

Veamos a continuación los datos recogidos del análisis de los textos escolares seleccionados. En relación con las sociedades actuales e históricas, si cuantificamos el indicador que con más frecuencia empleamos respecto al tipo de aprendizaje, el resultado es el Ind.C (aprendizaje memorístico de fechas, nombres, principios y teorías). Así se afirma en el libro del profesor en relación con el tema de La Edad Contemporánea de $6 .^{\circ}$, que «... todos los contenidos, que, además, son muy numerosos, son nuevos para ellos, lo que requerirá un gran esfuerzo para memorizarlos. Facilíteles la tarea repitiendo frecuentemente los nombres de los personajes que se mencionan y situándolos en el momento histórico que les corresponde» (Alzu y Henao, 2009b, p. 194).

También, en otros libros encontramos más ejemplos del Ind.C, al establecerse en la mayoría de las unidades un apartado denominado Resumo, donde a través de un mapa conceptual, la síntesis del tema se reduce a nombres, fechas y hechos. Igualmente se adscriben a este indicador actividades de refuerzo lo aprendido que establecen como contenidos mínimos para la 
unidad Vivimos en sociedad los siguientes: concepto de natalidad y mortalidad, conceptos de emigrante e inmigrante, población rural o urbana, evolución de la población en España. (Gómez, Valbuena y Brotons, 2009b, p. 198).

Sin embargo, también encontramos ejemplos, aunque en mucha menor medida, de aprendizaje escasamente relacionado con las ideas e intereses del alumnado (Ind.B). De este modo, en el tema de Pueblos y ciudades de 1. ${ }^{\circ}$ (Alzu y López Sáez, 2008) se le plantea al profesorado como sugerencia didáctica que al empezar «centre el interés de los alumnos en la fotografía del ayuntamiento y pregúnteles dónde se encuentra en su localidad. A continuación hágales preguntas para comprobar sus conocimientos sobre el ayuntamiento: ¿Quién se encarga de barrer las calles? ¿Cuándo son las fiestas de la localidad? ¿Quién las organiza?...»(p. 126). En el tema de la Prehistoria de 5. (Alzu y Henao, 2009a), se intenta atender a los intereses del alumnado al indicarle al profesorado que le pida a este en relación con unas fotografías «que elijan la que les parece más interesante y expliquen por qué» (p. 178). Este último ejemplo pone de manifiesto que se trata de un aprendizaje muy superficialmente relacionado con las ideas e intereses del alumnado, ya que se refiere a temas académicos y no a aspectos del entorno social o cultural del mismo. Además, obsérvese que solo es en el libro del profesor donde se propone a veces contextualizar las actividades en el entorno del alumnado o realizar otras actividades que establezcan esa relación con el medio próximo. Esta escasa relación con el entorno y los intereses del alumnado, también se observa en los textos de Expresión Plástica y Visual (Llorente, Andrieu, Montorio y Lekue, 2003).

Los datos de las entrevistas señalan una tendencia similar al análisis de los materiales, tanto en el caso de profesores que utilizan el libro de texto como de aquellos que elaboran materiales propios. Así, algunos docentes del CEIP V Centenario (Huelva) consideran que los libros de texto de Educación Infantil y Primaria promueven "un aprendizaje de memorieta y rutinario» $(6: 84,387: 387$, D3). Mientras que el aprendizaje que generan los materiales elaborados por ellos mismos se describen de la siguiente manera:

Se trata de un aprendizaje para que los niños actúen (6:85, 389:389, D5).

Más de razonamiento, para que los niños sean más protagonistas y puedan opinar, sin que se les invalide ninguna respuesta (6:85, 390:390, D3).

Por otra parte, en la práctica de maestros que no utilizan el libro de texto de forma habitual y elaboran materiales curriculares (MCEP), se insiste en la misma observación anterior: 
Cuando trabajamos sin libro de texto con un planteamiento común llegan los niños y niñas a $5 .^{\circ}$ y les planteas una unidad didáctica de cosas básicas que creen que conocen porque han tenido libros y porque han estudiado mucho, pues como puede ser en el caso nuestro, el estudio del río del pueblo que cruza el municipio y el siguiente. Pues ves que a pesar de todo lo que han estudiado, de todos los vídeos que han visto en $1 .^{\circ}, 2 .^{\circ}, 3 .^{\circ}$ y $4 .^{\circ}$, no tienen ni idea. Yo he comprobado este año en $5 .^{\circ}$ que una zona como la nuestra, que están todos los pueblos cercanos a recorrer en una hora en bicicleta, no había ningún niño o niña que los conociera todos. Es decir, tenemos claro que esos aprendizajes que se hacen en el libro, de los ríos o de las provincias de memoria, siguiendo lo que pone el libro, se olvida, y cuando llegan al tercer ciclo, esos aprendizajes ya no existen (7:31, 040:040, D4).

En cuanto a la función de los conocimientos previos, los resultados de nuestro análisis ponen de manifiesto que en la mayoría de los temas «los conocimientos personales solo se utilizan al comienzo del proceso de enseñanza-aprendizaje» (Ind.B). Así ocurre en el tema Las instituciones de España y de Andalucía de 5. (Alzu y Henao, 2009a, p. 159), donde aparecen tres preguntas que no se retoman a lo largo de su desarrollo. De este modo, se entiende el principio constructivista de "partir de los conocimientos previos de los alumnos» en un sentido temporal y no para facilitar la interacción entre saberes anteriores y el objeto de conocimiento (Aisenberg, 2000).

Además, esta utilización de las ideas previas de los estudiantes no es sistemática, ni tiene el mismo grado de importancia dependiendo del ciclo y del tema y, en la mayoría de las ocasiones, queda reducida a un mero repaso de lo estudiado en cursos anteriores. De este modo, el tema La historia de 2. ${ }^{\circ}$ (Herrero y Martín, 2010b) se enfoca desde lo más lejano en el tiempo, «la vida en las cavernas», "la vida en los castillos», hasta «la vida en la actualidad», preguntando al principio en Descubro lo que sé, no por los objetos o experiencias de la actualidad, sino: "colorea las personas que vivían en el castillo, rodea los objetos que usaban en el castillo ...»(p. 171), lo que responde a conocimientos escolares anteriores y no al conocimiento cotidiano.

También, respecto a esta categoría llama la atención que en el libro del profesor suele establecerse un apartado de dificultades de aprendizaje al comienzo de cada tema en el que se contemplan recomendaciones. Así en el tema 15 de $2 .^{\circ}$ El paso del tiempo (Alzu y Henao, 2010), se indica al profesor, en previsión de dificultades, que «el concepto de tiempo histórico es difícil de comprender para los alumnos de esta edad. Por ello, se recomienda trabajarlo de la manera más concreta posible, es decir, utilizando identificadores temporales, como son antes y ahora, partiendo siempre de la propia historia 
personal del alumno» (p. 156B); algo similar ocurre en el tema Notamos el paso del tiempo, de 3. ${ }^{\circ}$ (Gómez y Valbuena, 2009).

Sin embargo, en los siguientes temas de Historia, que suelen estudiarse en $5 .^{\circ}$ y $6 .^{\circ}$, ya se olvidan estas dificultades de aprendizaje y se comienza por la Prehistoria para continuar con la Edad Antigua y Media, la Edad Moderna, y finalmente, la Edad Contemporánea. Este enfoque cronológico de la enseñanza de la Historia, contradictorio con una perspectiva didáctica actualizada, continúa siendo indefectiblemente el canon de la secuenciación del conocimiento escolar. Se procede de igual manera con el espacio. En los primeros ciclos los temas intentan enlazar con el entorno: la calle, la casa, la familia, la Comunidad Autónoma; para en el último ciclo, no centrarse en estos trabajándolos en una mayor complejidad, sino en otros más «lejanos» como España y Europa, confundiendo proximidad con distancia territorial.

En la detección de estas dificultades de aprendizaje, por otra parte, no se alude a los resultados de la investigación didáctica y psicológica, sino que responden al más elemental sentido común, así en el tema La sociedad de Europa y de España de 6. (Gómez, et al., 2009a) se indica: «la mayor dificultad de estos contenidos, seguramente, se encontrará en las páginas dedicadas a la organización y gobierno de la UE, y ello se debe a la imposibilidad de simplificar dichos contenidos. No obstante, en el ámbito del aula puede ser más que suficiente hacer ver al alumnado que la UE mantiene el principio de la separación de poderes para garantizar la democracia en su gobierno» (p.196).

\section{CONCLUSIONES}

De los resultados de este estudio podemos extraer algunas consideraciones relevantes que planteamos a continuación de forma sintética y esquemática:

a) El conocimiento escolar que se maneja en los libros de texto referente a sociedades actuales e históricas y actividades económicas es, en gran medida, una copia reducida del conocimiento científico, si bien encontramos algunos ejemplos en el primer ciclo en los que se busca una mayor aproximación al conocimiento cotidiano del alumnado.

b) En cuanto a los aspectos axiológicos, aunque el propósito básico de los libros de texto analizados es aportar al alumnado los conocimientos necesarios para superar los cursos escolares, también tiene cierta importancia la finalidad cultural y de supuesto respeto por el medio, si bien en muchas ocasiones a modo de sugerencia, comentario o recomendación, se plantean en el libro del profesor. 
c) En lo referente a los aspectos psicológicos, hemos encontrado, respecto al tipo de aprendizaje que favorece el material, que se trata en la mayoría de los temas de un aprendizaje memorístico, principalmente de fechas y nombres, aunque también existen algunos ejemplos que tratan de favorecer un aprendizaje muy superficialmente relacionado con las ideas e intereses del alumnado, buscando la contextualización en el entorno del alumnado, si bien, de nuevo estas propuestas se plantean solo en el libro del profesor.

d) Los obstáculos que se observan en el conocimiento social, histórico y económico que trasmiten los materiales analizados, corroborados por las declaraciones del profesorado, son fundamentalmente que los libros de texto contribuyen escasamente a lograr una comprensión histórica y actualizada del funcionamiento social y económico de las sociedades.

La investigación didáctica ha demostrado que el manual escolar continúa siendo el material escolar mayoritario en la práctica de aula, sin embargo los resultados de nuestra investigación detectan obstáculos en tales manuales que dificultan la comprensión por el alumnado de las complejas sociedades actuales e históricas y de la actividad económica, por lo que es preciso acometer una labor de investigación e innovación didáctica y científica de los libros de texto que supere tales obstáculos. Se necesitan materiales curriculares para la enseñanza del Medio Social y Cultural que, en el aspecto epistemológico, propongan secuencias de conocimiento escolar basadas en la interacción del conocimiento cotidiano del alumnado en relación con lo social y el científico; en el aspecto axiológico, favorezcan actitudes y valores hacia un pensamiento práctico, reflexivo y crítico para contribuir a mejorar la realidad socioeconómica; y, en cuanto a los aspectos psicológicos, promuevan un aprendizaje significativo y relevante relacionando las ideas personales y los intereses del alumnado con las nuevas informaciones procedentes de fuentes diversas.

\section{NOTAS}

1 Este trabajo es resultado del Proyecto de Excelencia: ¿Cómo se realiza la enseñanza sobre la realidad social y natural en las aulas de Educación Infantil y Primaria de Andalucía? Estudio de las estrategias didácticas y propuestas de mejora. SEJ-5219 (2010-2014). Y del Proyecto I+D: ¿Cómo mejorar la enseñanza elemental sobre el medio?: análisis del currículo, los materiales y la práctica docente. EDU2009-12760EDUC (2009-2012).

2 Proyecto I+D SEJ2004-04962/EDUC: Un estudio sobre los obstáculos y dificultades didácticas del profesorado de primaria en el diseño y puesta en práctica de procesos de investigación escolar: elaboración y experimentación de una propuesta para la formación del profesorado». 
3 El cuestionario diseñado por el Proyecto SEJ-5219 y administrado durante los cursos 201011 y 2011-12 a una muestra de 863 docentes andaluces de 94 centros escolares, obtuvo entre otros los resultados siguientes: las editoriales utilizadas por el profesorado en su práctica de aula son: Anaya 29\%, Santillana $20.7 \%$, SM 10,7\%, Edelvives 5,1\%, Everest 3,4\% y Vicens Vives $3 \%$.

4 El proyecto INM (6-12) es un material didáctico que está organizado para facilitar la planificación y el desarrollo de la enseñanza de equipos de maestros de educación primaria interesados por las propuestas de investigación en el aula.

5 Como es sabido, Hot Potatoes son ejercicios educativos del tipo respuesta corta, selección múltiple, completar frases...

\section{AGRADECIMIENTOS}

Queremos expresar nuestra gratitud a los centros educativos y al profesorado que han colaborado en esta investigación y que se citan en el texto. 


\section{REFERENCIAS BIBLIOGRÁFICAS}

Alzu, J. L. y López Sáez. M. (2008). Conocimiento del medio 1. Sevilla: Santillana Educación.

Alzu, J. L. y Henao, J.T. (2009a). Conocimiento del medio 5. Sevilla: Santillana Educación

Alzu, J. L. y Henao, J. T. (2009b). Conocimiento del medio 6. Sevilla: Santillana Educación

Alzu, J. L. y Henao, J. T. (2010). Conocimiento del medio 2. Sevilla: Santillana Educación.

Aisenberg, B. (2000). Los conocimientos previos en situaciones de enseñanza de las Ciencias Sociales. En J. A. Castorina y A. M. Lenzi, La formación de los conocimientos sociales en los niños. Barcelona: Gedisa.

Atienza, E., y Van Dijk, T. A. (2010). Identidad social e ideología en libros de texto españoles de Ciencias Sociales. Revista de Educación, 353, 67-106.

Armento, B. (2003). Conceptos sobre el trabajo y aspiraciones de niños y niñas de un barrio del centro de la ciudad y de un barrio periférico. Enseñanza de las Ciencias Sociales: revista de investigación, 2, 13-26.

Barton, K. C. (2008). Research on student's ideas about history. En L.S.. Levstik \& C. A. Tyson (eds,), Handbook of Research in Social Studies Education (pp. 239-257). New York/London: Routledge.

Benejam, P. (2015). ¿Qué educación queremos? Barcelona: Octaedro.

Blanco, A. (2007). La representación del tiempo histórico en los libros de texto de primero y segundo de la enseñanza secundaria obligatoria. [Tesis doctoral]. Universidad de Barcelona.
Caba de la M. A., y López Atxurra, J. R. (2005). Actividades de participación y desarrollo de competencias de ciudadanía en los libros de texto de Educación Primaria de la Comunidad Autónoma Vasca (Conocimiento del medio). Revista de Educación, 336, 377-396.

Cañal, P., Travé, G., y Pozuelos, F. J. (2011). Análisis de los obstáculos y dificultades de profesores y estudiantes en la utilización de enfoques de investigación escolar. Investigación en la Escuela, 73, 5-26.

Carretero, M., y López, C. (2009). Estudios cognitivos sobre el conocimiento histórico: aportaciones para la enseñanza y alfabetización histórica. Enseñanza de las ciencias sociales: revista de investigación, 8, 75-89.

Delval, J. (2013). El descubrimiento del mundo económico en niños y adolescentes. Madrid: Morata.

Delval, J., Díaz Barriga, F., Hinojosa, M. L., y Daza, D. (2006). Experiencia y comprensión: concepciones sobre el trabajo en menores que trabajan en la calle en la Ciudad de México. Revista Mexicana de Investigación Educativa, 11(31), 1337-1362.

Díaz Castorina, J. A. (2010). Desarrollo del conocimiento social: prácticas, discursos y teoría. Buenos Aires: Miño y Dávila.

Díez, E. (2012). Children's understanding of commercial street ads and signs related to economic interchanges. En J. A. García Madruga et al. (Coords.) Construyendo Mentes: Homenaje a Juan Delval. Madrid: UNED

Domínguez, J., y Feito, R. (2007). Finalidades de la educación en una sociedad 
democrática: Alternativas para un sistema escolar democrático. Barcelona: Editorial Octaedro.

Estepa, J. (2003). Concepciones de los alumnos de primaria sobre el cambio en las sociedades. Una investigación empírica. Reseñas de Enseñanza de la Historia, 1, 213-226.

Estepa, J., Ferreras, M., López, I. y Morón, H. (2011). Análisis del patrimonio presente en los libros de texto: obstáculos, dificultades y propuestas. Revista de Educación, 355, 573-588. doi: 10-4438/1988-592X-RE-2011-355037

Estepa, J. (2012). La formación del profesorado para enseñar la participación en el Grado de Maestro y en el Máster en Profesorado de Secundaria. Una alternativa basada en los problemas prácticos profesionales, En N. de Alba, F. F. García Pérez y A. Santisteban (Eds.) Educar para la participación ciudadana en la enseñanza de las Ciencias Sociales (pp. 211-220). Sevilla: AUPDCCSS- Díada.

Etxebarria, L. et al. (2009). Conocimiento de Medio $6 .^{\circ}$ curso. Guía. Recursos didácticos para el profesorado. Proyecto: La casa del saber. Madrid: Santillana.

Fourez, G. (2008). Cómo se elabora el conocimiento. La epistemología desde un en-foque socioconstructivista. Madrid: Narcea.

García Pérez, F.F. (2003). Las ideas de los alumnos y la enseñanza del medio urbano. Sevilla: Díada.

García, P. y Leduc, J. (2003). L'enseignement de l'histoire en France de l'Ancien Régime á nos jour. París: Armand Colin.

Gómez, R. y Valbuena, R. (2009). Conocimiento del medio 3. Madrid: Grupo Anaya.
Gómez, R., Valbuena, R. y Brotons, J. R. (2009a). Conocimiento del Medio 6. Madrid: Anaya.

Gómez, R., Valbuena, R. y Brotons, J. R. (2009b). Conocimiento del medio 5. Madrid: Grupo Anaya.

Hernández, A. (2012). ¿Cómo tratan los libros de texto de bachillerato la crisis económica? Análisis de contenido. Investigación en la escuela, 76, 51-64.

Herrero, M. y Martín, M. G. (2010a). Conocimiento del medio 1. Madrid: Grupo Anaya.

Herrero, M. y Martín, M. G. (2010b). Conocimiento del medio 2. Madrid: Grupo Anaya.

Houser, N. O. \& Kuzmic, J. J. (2001). Ethical Citizenship in a Postmodern World: Toward a More Connected Approach to Social Education for the Twenty-First Century. Theory and Research in Social Education, 29(3), 431461. doi:10.1080/00933104.2001.1050 5950

Levesque, S. (2008). Thinking Historically, Educating Students for the TwentyFirst Century. Canada: University of Toronto.

Llorente, E., Andrieu, A., Montorio, A., y Lekue, P. (2003). Análisis de libros de texto de Expresión Plástica y Visual de Educación Primaria. Revista de Psicodidáctica, 14, 149-160. doi: http://dx.doi.org/10.1387/RevPsicodidact.151

McDonough, K. \& Feinberg, W. (Eds.) (2003). Education and citizenship in liberal-democratic societies: teaching for cosmopolitan values and collective identities. Oxford: Oxford University Press.

Mckernan, J. (1999). Investigación-acción y currículum. Madrid: Morata 
Miller, S., y Vanfossen, P. J. (2008). Recent research on the teaching and learning of pre collegiate economics. In L. S. Levstik and C. A. Tyson. Handbook of Research in Social Studies Education. New York/ London: Routledge.

Nicholls, J. (2003). Methods in School Textbook Research. International Journal of Historical Learning, Teaching and Research, 3(2), 1-17.

Niemi, R. G., y Junn, J. (1998). Civic Education: What Makes Students Learn. New Haven: Yale University Press.

Osborne, K. (1991). Teaching for democratic citizenship. Montreal: Our Schools.

Pagès, J. y Santisteban, A. (Coords.) (2011). Didáctica del Conocimiento del Medio Social y Cultural en la Educación Primaria. Madrid: Síntesis.

Porlán, R. y Rivero, A. (1998). El conocimiento de los profesores. Una propuesta formativa en el área de ciencias. Sevilla: Díada.

Posnanski, T. J., Schug, M. C., y Schmitt, T. (2006). Can Students Learn Economics and Personal Finance in a Specialized Elementary School? International Journal of Social Education, 21(2), 196-205.

Pozuelos, F. J., Travé, G. y Cañal, P. (2007). Acerca de cómo el profesorado de primaria concibe y experimenta los procesos de investigación escolar. Revista de Educación, 344, 403-423.

Pozuelos, F. J., Travé, G. y Cañal, P. (2010). 'Inquiry-based teaching: teachers' conceptions, impediments and support', Teaching Education, 21(2), 131-142. doi: $10.1080 / 10476210903494507$

Prats, J. y Santacana, J. (2011). Por qué y para qué enseñar historia. En J. Prats
(Coord.), Didáctica de la Geografía y la Historia. Barcelona: Graó.

Rodríguez, M. y Carretero, M. (2012). El cambio conceptual en historia: influencias piagetianas y tendencias actuales. En J. A. García Madruga et al. (Coords.), Construyendo Mentes: Homenaje a Juan Delval. Madrid: UNED.

Santisteban, A. (2007). Una investigación sobre cómo se aprende a enseñar el tiempo histórico. Enseñanza de las Ciencias Sociales, 6, 19-29.

Simons, H. (2011). El estudio de casos. Teoría y práctica. Madrid, Morata.

Sobejano, M. J. y Torres, P. A. (2009). Enseñanza de la historia en secundaria. Historia para el presente y educación ciudadano. Madrid: Tecnos.

Solaz-Portolés, J. J. (2010). La naturaleza de la Ciencia y los libros de texto de ciencias: una revisión. Educación $X X I, 13(1), 65-80$.

Travé, G., y Delval, J. (2009). Análisis de la práctica de aula. El caso de las concepciones histórico-económicas del alumnado. Investigación en la Escuela, $69,5-17$.

Travé, G. y Pozuelos, F. J. (2008). Consideraciones didácticas acerca de las líneas de investigación en materiales curriculares. A modo de presentación. Investigación en la Escuela, 65, 3-10.

Travé, G., Pozuelos, F. J., Cañal, P. y De las Heras, M. A. (2013). Experimentación de una guía de análisis de materiales y desarrollo de la enseñanza del medio natural y social. Investigación en la Escuela, 81, 5-20

Valls, R. (2007). Historiografía escolar española: siglos XIX-XXI. Madrid: UNED. 


\section{PERFIL ACADÉMICO Y PROFESIONAL DE LOS AUTORES}

Gabriel Travé. Doctor en Ciencias de la Educación y Profesor Titular de Didáctica de las Ciencias Sociales, Universidad de Huelva. Ha sido profesor de Educación Primaria, Secundaria y Asesor del Centro del Profesorado de Huelva. En cuanto a la actividad investigadora, destacan las líneas siguientes: materiales curriculares, investigación escolar, Didáctica de la Economía, así como formación inicial y desarrollo profesional del profesorado de Ciencias Sociales.

Jesús Estepa. Catedrático de Universidad de Didáctica de las Ciencias Sociales desde 2004, profesor de esta disciplina en la formación inicial de maestros de Educación Primaria y de profesorado de Ciencias Sociales, Geografía e Historia de Educación Secundaria, nivel educativo en el que fue profesor durante diez años. Sus principales líneas de investigación son: formación inicial y desarrollo profesional del profesorado de Ciencias Sociales, Educación Patrimonial, Didáctica de la Historia y Educación para la Ciudadanía.

Juan Delval. Doctor en Filosofía por la Universidad Complutense de Madrid, posee una dilatada trayectoria profesional: Catedrático de Psicología Evolutiva en las universidades Autónoma y Complutense de Madrid y UNED. Ha pronunciado conferencias, impartido cursos y realizado trabajos de investigación en diversos países del ámbito iberoamericano. Ha sido representante español en diversas reuniones internacionales de la UNESCO, OCDE, Consejo de Europa, etc. Y es miembro del Consejo Asesor de importantes revistas de Psicología y Educación.

Dirección de los autores: Gabriel Travé
Jesús Estepa
Universidad de Huelva
Departamento de Didácticas Integradas
Facultad de Ciencias de la Educación
Campus de El Carmen
Avda. Fuerzas Armadas, s/n
21071 Huelva
E-mail: trave@uhu.es
jestepa@uhu.es
Juan Delval
Facultad de Educación
Universidad Nacional de Educación a Distancia
C/ Juan del Rosal, 14
28040 - Madrid
E-mail: jdelval@psi.uned.es
juan.delval@uam.es 
Fecha Recepción del Artículo: 05. Febrero. 2014

Fecha modificación Artículo: 31. Marzo. 2014

Fecha Aceptación del Artículo: 02. Abril. 2014

Fecha Revisión para Publicación: 13. Junio. 2016 